\title{
Rhodnius zeledoni sp. nov. afim de Rhodnius paraensis Sherlock, Guitton \& Miles, 1977 (Hemiptera, Reduviidae, Triatominae)
}

\author{
José Jurberg ${ }^{1,2}$, Dayse da Silva Rocha ${ }^{1}$ \& Cleber Galvão ${ }^{1}$ \\ ${ }^{1}$ Laboratório Nacional e Internacional de Referência em Taxonomia de Triatomíneos, \\ Instituto Oswaldo Cruz, \\ Av. Brasil, 4365, Manguinhos, CEP 21045-900, Rio de Janeiro, Brasil \\ ${ }^{2}$ Autor p/ correspondência: José Jurberg, e-mail: jjurberg@ioc.fiocruz.br
}

JURBERG, J., ROCHA, DS. \& GALVÃO, C. Rhodnius zeledoni sp. nov. afim de Rhodnius paraensis Sherlock, Guitton \& Miles, 1977 (Hemiptera, Reduviidae, Triatominae). Biota Neotrop., 9(1): http://www. biotaneotropica.org.br/v9n1/en/abstract?article+bn02509012009.

Abstract: A new species of Rhodnius is described and illustrated from a male specimen from northeastern Brazil. The specimen was found died and dried. This species can be distinguished from its similar congener R. paraensis Sherlock, Guitton \& Miles by the greater size, the general aspect of the color of the body, by the uniform color of the legs, different proportions between the morphological structures of the head and the segments of the rostrum; also, in the male genitalia by the aspect of median process of pygophore, dorsal phallotheca plate and phallotheca process.

Keywords: Chagas disease, systematic, Rhodnius zeledoni sp. nov., Hemiptera, taxonomy.

JURBERG, J., ROCHA, DS. \& GALVÃO, C. Rhodnius zeledoni sp. nov. afim de Rhodnius paraensis Sherlock, Guitton \& Miles, 1977 (Hemiptera, Reduviidae, Triatominae). Biota Neotrop., 9(1): http://www. biotaneotropica.org.br/v9n1/pt/abstract?article+bn02509012009.

Resumo: Uma nova espécie de Rhodnius é descrita e ilustrada baseada em um exemplar macho do nordeste brasileiro. O espécime foi encontrado morto e ressecado. Esta espécie pode ser separada da espécie afim, R. paraensis Sherlock, Guitton \& Miles, pelo seu maior tamanho, pelo aspecto geral de coloração do corpo, pela cor uniforme das pernas, pelas diferentes proporções entre as estruturas morfológicas da cabeça e dos segmentos do rostro; ainda, na genitália masculina, pelo aspecto do processo mediano do pigóforo, do falosoma e de seu processo.

Palavras-chave: doença de Chagas, sistemática, Rhodnius zeledoni sp. nov., Hemiptera, taxonomia. 


\section{Introdução}

Desde que o Laboratório de Triatomíneos do Instituto Oswaldo Cruz se tornou Centro de Referência do Ministério da Saúde em 1989 um propício relacionamento entre as partes tem promovido inúmeras cooperações técnico-científico, entre elas destaca-se a identificação de exemplares coletados cujas características morfológicas não correspondem à fauna reconhecida das áreas trabalhadas pela Secretaria de Vigilância Sanitária. Este fato tem propiciado a descoberta de inúmeras espécies novas (Jurberg 1999).

Em março de 2007 recebemos para identificação do "PtoEmolacen" de Aracajú-Sergipe, dois triatomíneos do gênero Rhodnius Stål, 1859, que foram coletados mortos e ressecados. Em uma análise verificamos que um deles era brethesi, Matta, 1919 e o outro não se enquadrava nas descrições de nenhuma das dezesseis espécies conhecidas do gênero. (Lent \& Wygodzinsky 1979, Galvão et al. 2003, Jurberg \& Galvão 2006).

As espécies do gênero Rhodnius tem padrões definidos em relação a biologia pois a maioria das espécies são silvestres; vivem em folhagens de palmeiras, buracos de árvores, sugando aves, roedores, morcegos e marsupiais, colocam seus ovos aderidos ao substrato o que favorece a sua dispersão, passiva e ativa, pois muitas aves que nidificam nas palmeiras tem hábitos migratórios. Ainda podem ser reconhecidas pela morfologia externa com seu tamanho reduzido e o tubérculo antenífero localizado no ápice da cabeça, genitália masculina caracterizada pela ausência de suporte do falosoma, na maioria das espécies, pelo formato do processo mediano do pigóforo, pela coloração homogênea tendendo sempre para castanho claro/escuro, com manchas mais ou menos acentuadas, e pela morfologia e ornamentação dos ovos. (Lent \& Jurberg 1969, Lent \& Wygodzinsky 1979, Barata 1981, 1998, Lent, Jurberg \& Galvão 1993, Jurberg 1995, Jurberg, Lent \& Galvão 1998, Carcavallo et al. 1998, 1999, Mejia, Galvão \& Jurberg, 1999, Jurberg et al., 2005, Jurberg \& Galvão 2006.

Carcavallo et al. (2000) definiram para o gênero Rhodnius Stål quatro complexos a saber: "Complexo Rhodnius pictipes Stål, 1872 com duas espécies: $R$. pictipes e $R$. stali Lent, Jurberg \& Galvão 1993, Complexo Rhodnius prolixus Stål 1859 com cinco espécies: $R$. prolixus, $R$. domesticus Neiva \& Pinto 1923, R. nasutus Stål 1859, R. neglectus Lent, 1954 e R. robustus Larrousse, 1927; Complexo $R$. pallescens Barber, 1932 com três espécies: R. pallescens, R. colombiensis Mejia, Galvão \& Jurberg, 1999 e R. ecuadoriensis Lent \& Leon, 1958; Complexo R. dalessandroi Carcavallo \& Barreto, 1976 com duas espécies: $R$. dalessandroi e R. milesi Carcavallo, Rocha, Galvão \& Jurberg.

As outras quatro espécies não possuíam caracteres morfológicas suficientes para constituir complexos, a saber: $R$. amazonicus Santos \& Sposina, 1973, $R$. brethesi Matta, 1919; $R$. neivai Lent, 1953 e $R$. paraensis Sherlock, Guitton \& Miles, 1977. Esta nova espécie aqui descrita $R$. zeledoni tem características morfológicas assemelhadas a $R$. paraensis.

\section{Resultados}

\section{Rhodnius zeledoni sp. nov.}

Material Tipo: Holótipo coletado por Dra. Catarina Zita Dantas de Araújo em Aracaju- SE e depositado na Coleção Herman Lent - Instituto Oswaldo Cruz/Fiocruz sob o n ${ }^{\circ} 3078$.

Macho - comprimento total $13 \mathrm{~mm}$, Pronoto: largura na região umeral $3 \mathrm{~mm}$, na região do colar $1 \mathrm{~mm}$, comprimento $2 \mathrm{~mm}$; Escutelo: comprimento $2 \mathrm{~mm}$; Abdômen: largura $5 \mathrm{~mm}$.
Cor geral castanho claro, intercalada com áreas castanho mais escuras em algumas regiões da cabeça, do hemiélitro e do abdômen (Figura 1).

Cabeça vista dorsalmente de cor castanho claro representada por uma larga faixa que vai do pescoço até o anti-clípeo. Lateralmente de cor castanho mais escuro que abrange a região do pescoço até o ápice das genas incluindo o tubérculo antenífero e o $1^{\circ}$ segmento antenal. Toda a superfície da cabeça esta recoberta por pequenos tubérculos com cerdas claras decumbentes que fornece um aspecto granuloso para a superfície. (Figuras 1,2,3)

Cabeça mais longa que larga ao nível dos olhos, relação 1:0,36; cabeça mais longa que o pronoto 1:0,69; região ante-ocular mais longa que a pós-ocular, relação 1:0,32. Região pós-ocular globosa separada do pescoço por uma constrição, o que lhe confere um aspecto de ampulheta. Genas de forma oblonga, aguçada no ápice ultrapassando levemente o clípeo. Clípeo de ápice afilado (Figuras 2 e 3).

Olhos medianos, bordo anterior arredondado, posterior retilíneo, inferior ultrapassando o limite da cabeça (Figura 3).

Relação entre a largura de um olho e a região inter-ocular (sinlipsis) 1:1,27.

Ocelos pequenos situados látero-dorsalmente logo após uma pequena depressão dentro da área escurecida que emerge da região lateral da cabeça, as áreas escuras ultrapassam a região pós-ocular e vão até próximo ao pescoço (Figuras 2 e 3 ).

Antena: primeiro segmento castanho escuro, não ultrapassando as genas, comprimento 0,25 mm. (Figuras 1,2,3), demais segmentos ausentes.

Rostro: primeiro segmento muito curto atingindo o nível mediano do tubérculo antenífero, segundo segmento muito longo mais de três vezes o tamanho do primeiro, atingindo o nível do

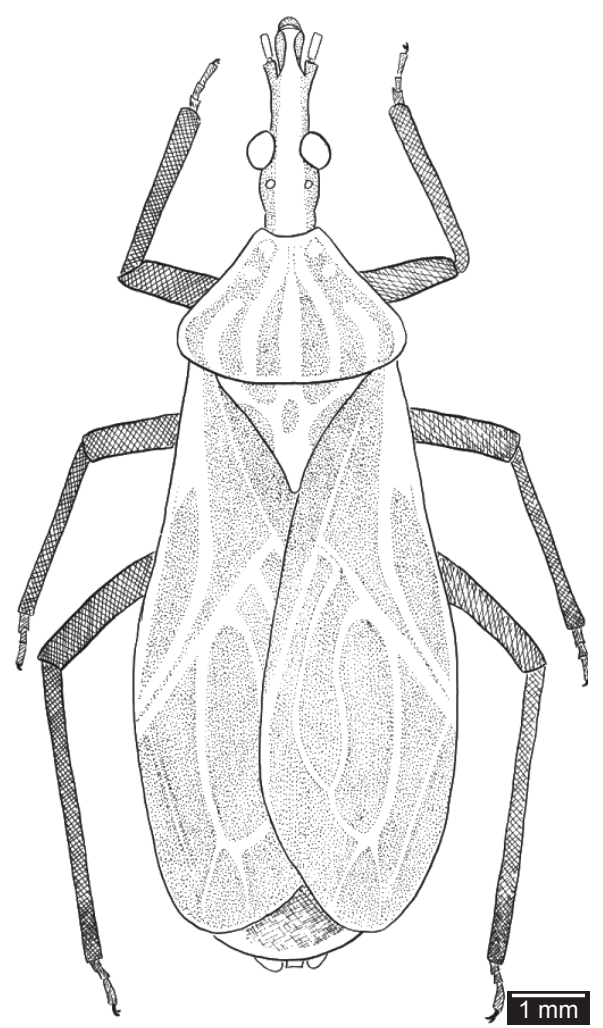

Figura 1. Rhodnius zeledoni sp. nov. macho vista dorsal.

Figure 1. Rhodnius zeledoni n. sp. male dorsal view. 
anel de constrição entre a cabeça e o pescoço, terceiro segmento muito curto menor que o primeiro atingindo o bordo anterior do sulco estridulatório. Relação entre os segmentos do rostro 1:3,15:0,84 (Figura 3).

Pronoto: castanho escuro, entremeando com $2+2$ carenas laterais castanho claro alaranjado e uma carena mediana com esculturação clara, todo o pronoto é circundado por uma faixa alaranjada. As carenas diferem entre si, as $1+1$ externas não são contínuas, as $1+1$ internas são contínuas. Lobo anterior do pronoto pouco diferenciado do lobo posterior, tubérculo discal e lateral inaparentes, como também o ângulo ântero-lateral e o colar (Figuras 1,2 e 5a).

Escutelo: triangular, castanho alaranjado nas carenas que o delimitam com $2+2$ depressões, um par lateral e outro par na
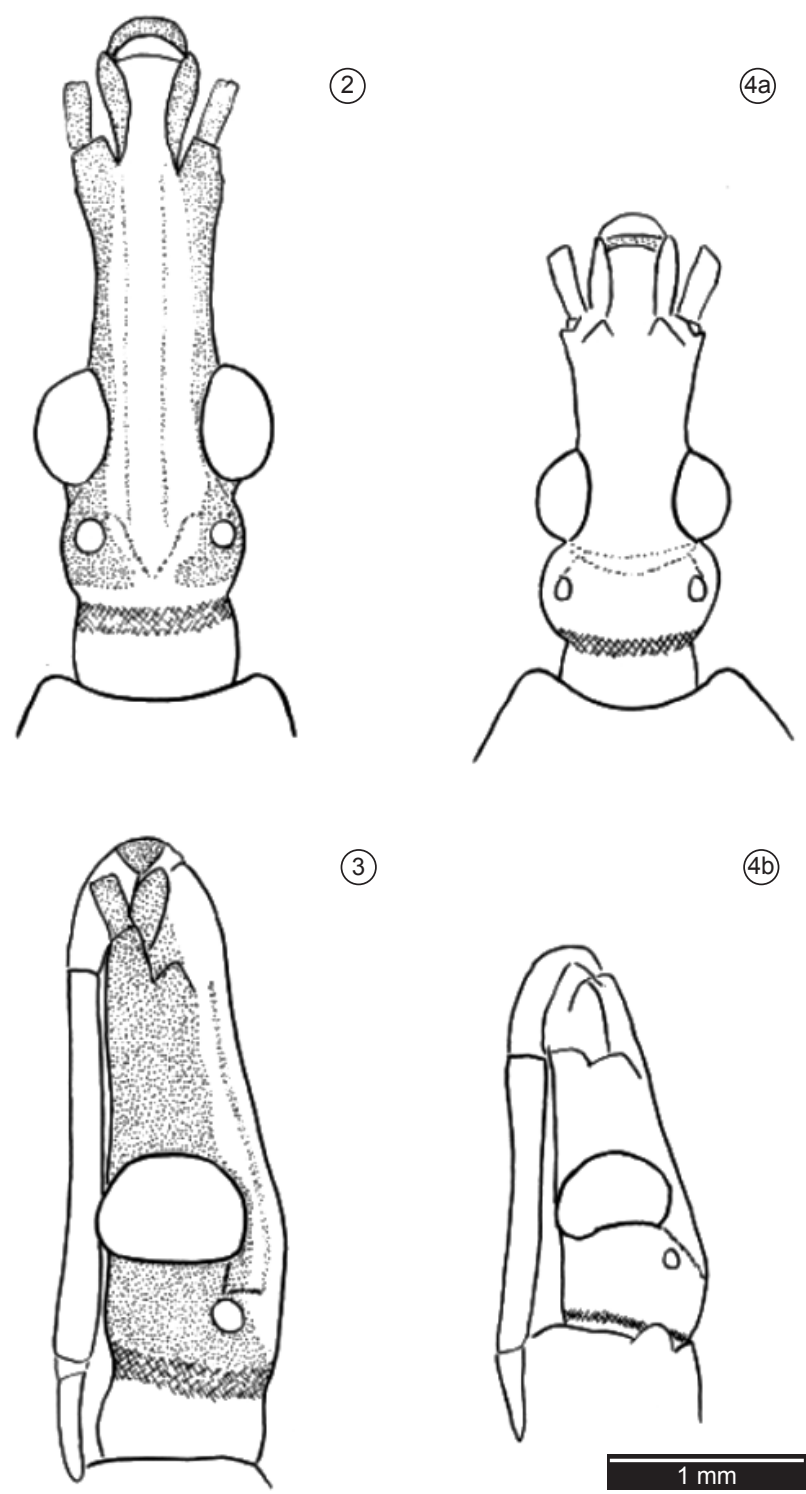

(3)
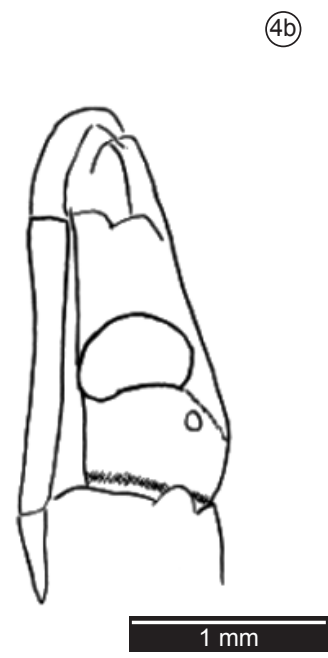

Figuras 2-4. Rhodnius zeledoni sp. nov. 2) cabeça vista dorsal; 3) cabeça vista lateral; 4a) Rhodnius paraensis Sherlock, Guitton \& Miles, 1977 cabeça vista dorsal, e 4b) Rhodnius paraensis vista de perfil.

Figures 2-4. Rhodnius zeledoni n. sp. 2) head dorsal view 3) head lateral view; 4a) Rhodnius paraensis Sherlock Guitton \& Miles, 1977 head dorsal view and 4b) Rhodnius paraensis lateral view. base do escutelo; na região central localiza-se uma depressão de forma ovóide. Processo do escutelo com ápice arredondado. Proporção entre a largura na base e o comprimento 1:0,85 (Figuras 1 e 5b).

Hemiélitros de coloração castanho alaranjado com as nervuras mais claras; base do corium e o clavo castanho escuros; recobrem o abdômen sem ultrapassar o ápice (Figura 1).
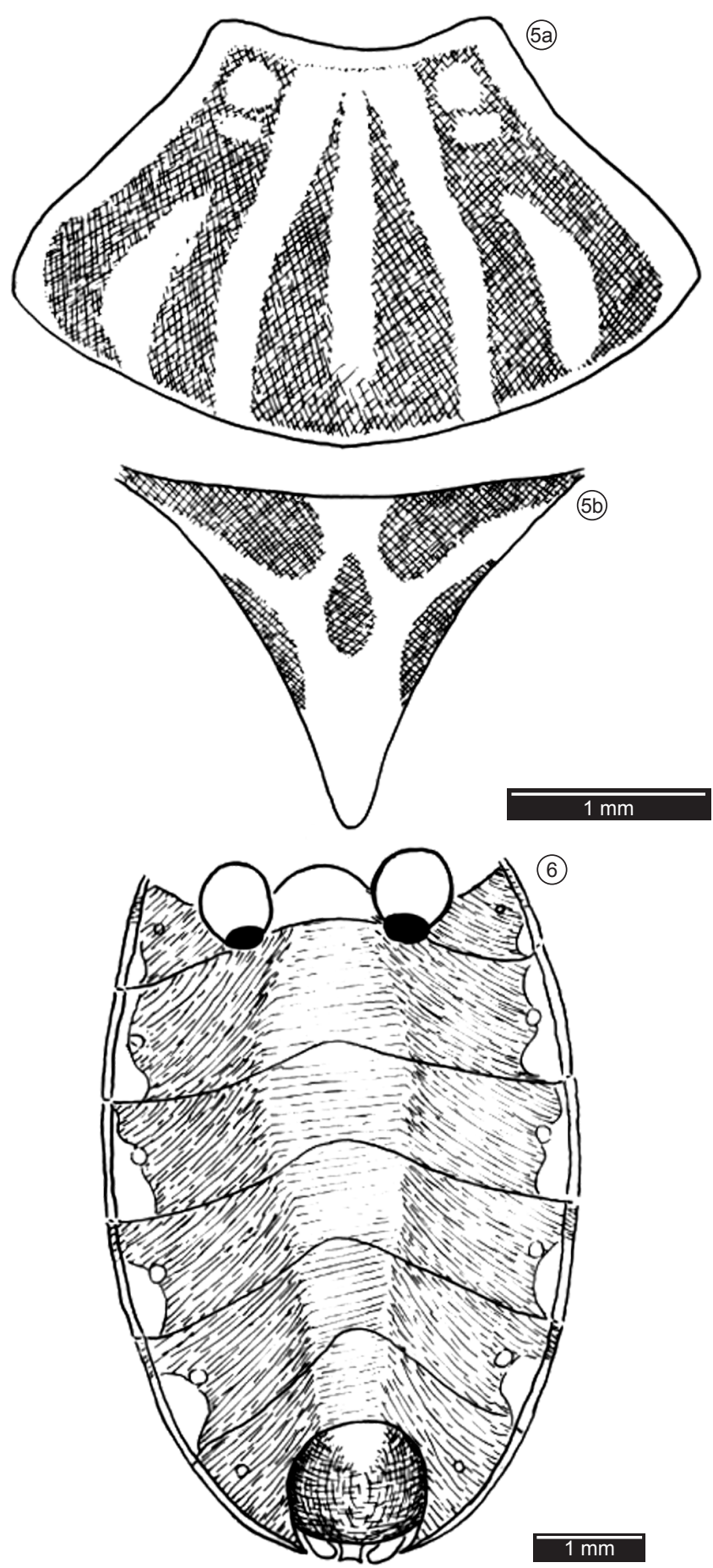

Figuras 5-6. Rhodnius zeledoni sp. nov. 5) Pronoto; 5b) escutelo, e 6) abdômen vista ventral.

Figures 5-6. Rhodnius zeledoni n.sp. 5) pronotum 5b) scutellum and 6) abdomen ventral view. 
Pernas: Nos três pares de pernas as coxas apresentam-se castanho escuro, os trocânteres castanho claro, fêmur castanho escuro, tíbias e tarsos do $1^{\circ}$ e $2^{\circ}$ par castanho escuro, tíbias do $3^{\circ}$ par castanho claro com exceção de anel apical que é escuro (Figura 1).

Abdômen ventralmente convexo de cor castanho escuro, tendo a região mediana uma faixa achatada mais clara que vai do $2^{\circ}$ urotergito até o bordo posterior do pigóforo. Lateralmente onde os urotergitos se soldam ao conexivo existem $1+1$ áreas claras do $2^{\circ}$ ao $7^{\circ}$ segmento abdominal, no $2^{\circ}$ segmento a área clara ocupa $1 / 3$ posterior do urotergito, no $3^{\circ}$ e $4^{\circ}$ segmentos ocupa toda a largura, com formato irregular atingindo os estigmas, no $5^{\circ}$ e $6^{\circ}$ a área é semi-circular e ocupa $2 / 3$ do segmento, $7^{\circ}$ segmento sem mancha clara.

Conexivo: não pode ser caracterizado por estar encoberto totalmente pelas asas do único exemplar examinado.

Genitália do macho constituída pelo $8^{\circ}$ e $9^{\circ}$ segmentos, este último denominado de Pigóforo (IX), constituído de uma cápsula ovóide, bastante quitinizada, com a base mais estreita e o ápice mais alongado, face ventral recoberta com pelos longos na região central envolvido por pêlos curtos, tendo no bordo posterior uma projeção com bordas laterais e apical levemente deprimidas, o denominado Processo Mediano do Pigóforo (PrP), visto ventralmente, castanho escuro nas áreas laterais e clara na faixa central (Figura 7).

Dentro do Pigóforo estão localizados o falo e se exteriorizando $1+1$ Parâmeros.

Os parâmeros $(\mathrm{Pa})$ são robustos cilíndricos, arqueados com 1/3 apical curvados tendo no seu ápice uma projeção triangular, a face dorsal e os bordos internos e externos com numerosas cerdas. (Figuras 8 e 9).

Falo (P) constituído por um aparelho articular (Apb) e o edeago (Ae) de proporções idênticas, quando distendido constata-se que a placa basal (Plb) é quatro vezes maior do que a Extensão mediana da placa basal (EPlb) com formato quadrangular. (Figuras 10, 11 e 12).

$\mathrm{O}$ edeago (Ae) se distingue por apresentar externamente um falosoma $(\mathrm{Ph})$ de forma ovóide, mostrando internamente um
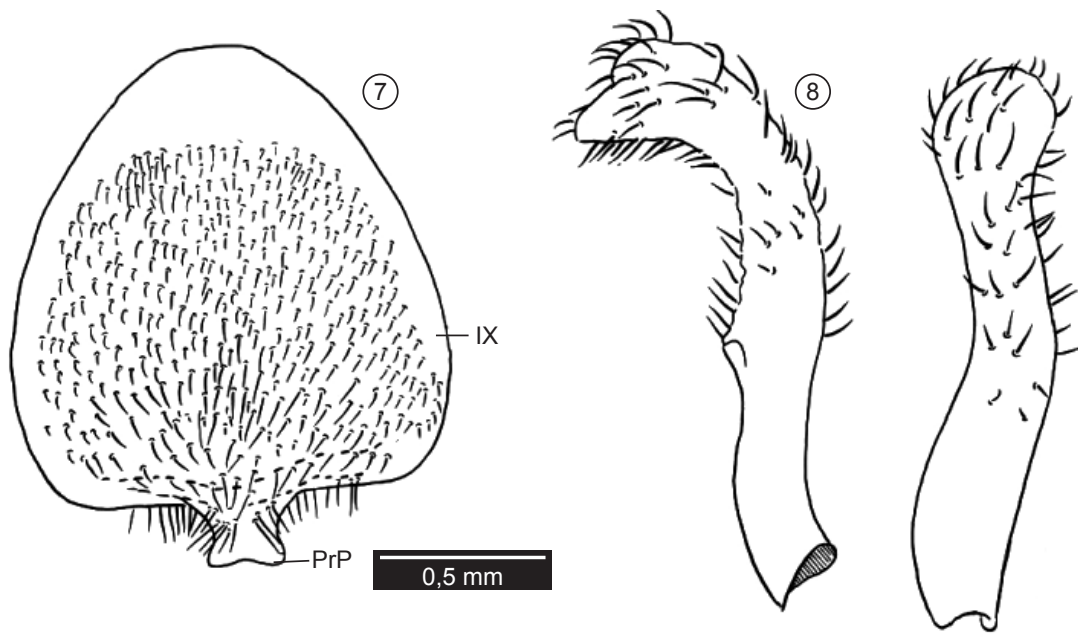

$0,3 \mathrm{~mm}$

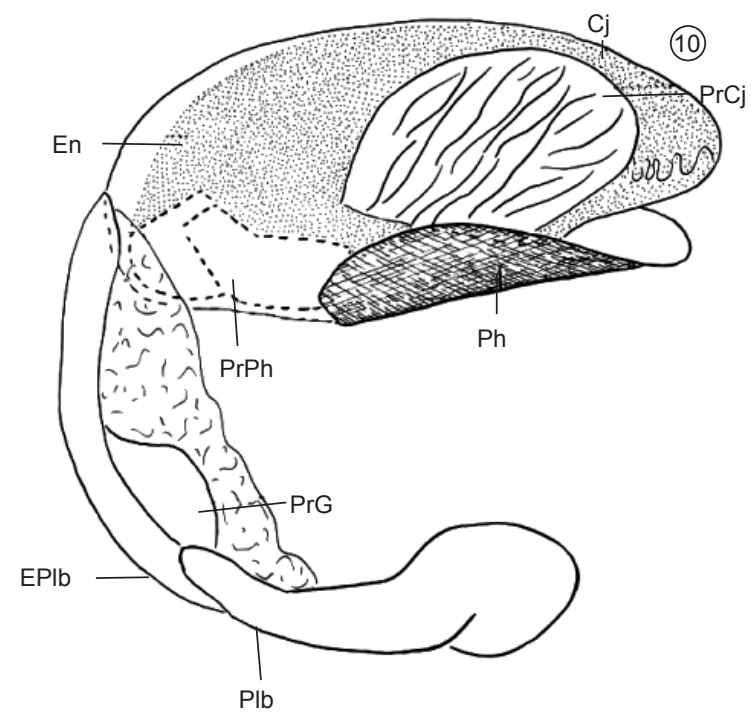

Figuras 7-10. Rhodnius zeledoni sp. nov. 7) Pigóforo vista dorsal; 8 e 9) Parâmeros vista dorsal e de perfil; e 10) Falo visto de perfil $($ Cj = conjuntiva; Em = Endosoma; $\mathrm{EPlb}=$ Extensão mediana da Placa basal, $\mathrm{Ph}=$ falosoma, $\mathrm{Plb}=$ Placa basal, $\mathrm{PrG}=$ Processo gonoporo; $\mathrm{PrPh}=\mathrm{Processo}$ do falosoma).

Figures 7-10. Rhodnius zeledoni $\mathrm{n} . \mathrm{sp}$. 7) pygophore dorsal view; 8 and 9) parameres dorsal view and lateral view; and 10) phallus lateral view (Cj conjunctive; En endosome; EPlb median extension of basal plate; Ph phallosome; Plb basal plate; PrG gonopore process , $\operatorname{PrPh}$ phallosome process). 

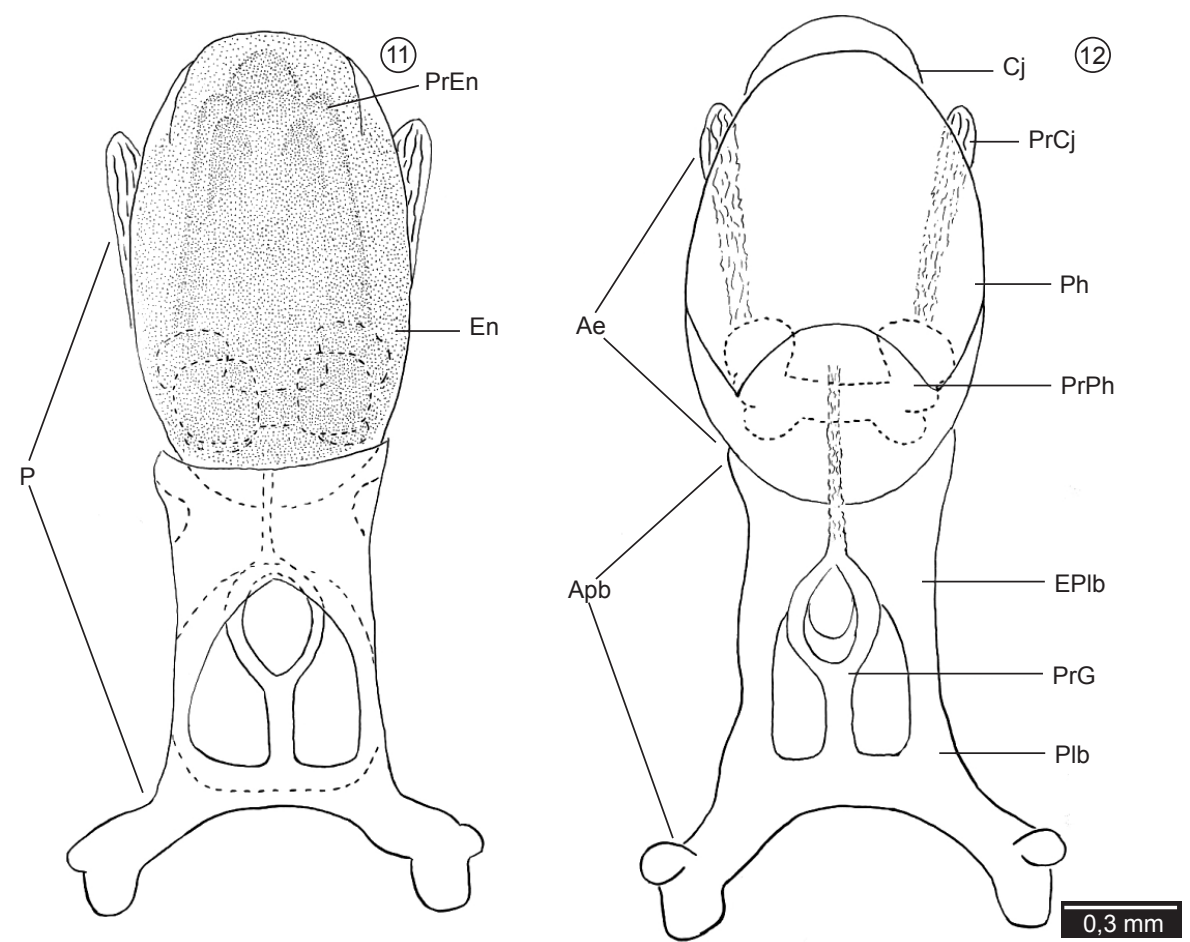

Figuras 11-12. Rhodnius zeledoni $\mathrm{sp}$. nov. 11) falo distendido vista dorsal, e 12) idem vista ventral. $\mathrm{Ae}=$ edeago; $\mathrm{Apb}=$ aparelho articular; $\mathrm{Cj}=$ conjuntiva; En = endosoma; $\mathrm{Eplb}=$ extensão mediana da placa basal $\mathrm{P}=$ falo $; \mathrm{PrEn}=$ processo do endosoma $\mathrm{Plb}=$ placa basal $; \mathrm{PrG}=$ processo do gonoporo $; \mathrm{PrPh}=$ processo do falosoma; $\mathrm{Ph}=$ falosoma, $\mathrm{PrCj}=$ processo da conjuntiva.

Figures 11-12. Rhodnius zeledoni n. sp. 11) phallus dorsal view, and 12) phallus ventral view. Ae aedeagus; $\mathrm{APb}$ articular apparatus; $\mathrm{Cj}$ conjunctive; En endosome; EPlb median extension of basal plate; Ph phallus; PrEn endosome process; Plb basal plate; PrG gonopore process; PrPh phallosome process; Ph phallosome; PrCj conjunctive process.

Tabela 1. Caracteres morfológicos diferenciais entre machos de Rhodnius zeledoni sp. nov. e Rhodnius paraensis Sherlock, Guitton \& Miles, 1997.

Table 1. Morphological differences between males of Rhodnius zeledoni n.sp. and Rhodnius paraensis Sherlock, Guitton \& miles, 1997.

\section{Rhodnius zeledoni sp. nov.}

\section{Rhodnius paraensis}

Comprimento

Proporção entre o comprimento da

cabeça e o pronoto

Largura máxima do pronoto

Proporção: comprimento da cabeça $x$

largura ao nível dos olhos

Proporção: largura do olho

Proporção: região ante-ocular X pós-ocular

Proporção entre os artículos do rostro

Antenas

Pernas

\section{$13 \mathrm{~mm}$ \\ $1: 0,69$}

$3 \mathrm{~mm}$

1:0,36

$1: 1,27$

$1: 0,32$

$1: 3,15: 0,84$

castanho escuro

Todas as pernas castanho escuras, sem manchas.
(Segundo Sherlock, Guitton \& Miles 1997)

$10.5-11 \mathrm{~mm}$
$1: 1$
$2.8 \mathrm{~mm}$
$1: 0,55$

$1: 1,55$
$1: 0,65$
$1: 2,7: 0,9$
castanho claro

Pernas anteriores menores, coxa escura, trocanter claro, fêmur escuro com mancha clara mediana e no ápice; tíbia com mancha clara na articulação com fêmur, tarsos escuros. (Figura 272 Lent \& Wygodzinsky 1979)

Tabela 2. Caracteres fálicos diferenciais entre Rhodnius zeledoni sp.nov. e Rhodnius paraensis Sherlock, Guitton \& Miles, 1977.

Table 2. Phalic structures differences between Rhodnius zeledoni n.sp. and Rhodnius paraensis Sherlock, Guitton \& miles, 1997.

\begin{tabular}{lcc}
\hline & Rhodnius zeledoni sp. nov. & $\begin{array}{c}\text { Rhodnius paraensis } \\
\text { (Segundo Sherlock, Guitton \& Miles) }\end{array}$ \\
\hline $\begin{array}{l}\text { Processo mediano do Pigóforo } \\
\text { (PrP) }\end{array}$ & $\begin{array}{l}\text { Retangular, com bordas laterais e apical } \\
\text { levemente deprimidas formando apicalmente } \\
\text { pontas arredondadas. (Figura 14) }\end{array}$ & $\begin{array}{l}\text { Retangular com bordas laterais e apical } \\
\text { levemente deprimidas formando } \\
\text { apicalmente, pontas afiladas. (Figura 13) } \\
\text { Ovóide }\end{array}$ \\
$\begin{array}{l}\text { Falosoma (Ph) } \\
\text { Processo do falosoma (PrPh) }\end{array}$ & em forma de alteres (Figura 12) & $1+1$ estrutura quitinizada \\
$\begin{array}{l}\text { Proporção entre o aparelho } \\
\text { articular (Apb) e a Extensão } \\
\text { mediana da placa basal (EPlb) }\end{array}$ & $1: 0,26$ & $1: 0,7$ \\
\hline
\end{tabular}



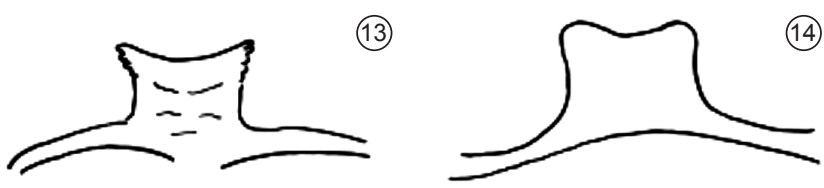

Figuras 13-14. Processo mediano do pigóforo, (PrP) - 13) R. zeledoni sp. nov.; e 14) R. paraensis.

Figures 13-14. median process of the pygophore; (PrP) - 13) R. zeledoni sp. $\mathrm{n}$.; and 14) R. paraensis.

processo do falosoma ( $\mathrm{PrPh}$ ) bastante quitinizada em forma de alteres; internamente preso ao endosoma (En) elástico estão delineados seus processos (PrEn). (Figuras 10, 11 e 12)

Recobrindo todo o endosoma a conjuntiva $(\mathrm{Cj})$ apresenta uma projeção membranosa, estriado bastante desenvolvido e o processo da Conjuntiva ( $\mathrm{PrCj})$.

Etimologia: esta espécie é dedicada ao Dr. Rodrigo Zelédon, famoso parasitologista nascido na Costa Rica que há muitos anos dedica-se ao estudo dos Triatomíneos, vetores da doença de Chagas tendo publicado dezenas de artigos científicos envolvendo os triatomíneos.

\section{Discussão}

A espécie que mais se assemelha a $R$. zeledoni sp.n é $R$. paraensis, pelo seu tamanho e coloração, porém dela se distinguindo pelo comprimento da cabeça, proporções comprimento da cabeça $x$ largura entre os olhos, região ante ocular e pós-ocular, artículos do rostro, coloração das pernas e por quatro estruturas fálicas: processo mediano do pigóforo, falosoma, Processo do falosoma, proporção entre aparelho articular e a extensão mediana da placa basal. (Tabelas 1 e 2) (Figuras 2,3, 4a, 4b).

\section{Agradecimentos}

Ao Serviço de Vigilância Sanitária do Ministério da Saúde na pessoa da Sra. Dra. Catarina Zita Dantas de Araújo da Gerência Executiva de Entomologia em Aracajú - Sergipe, por nos ter enviado os dois exemplares, auxílio do Conselho Nacional de Desenvolvimento Tecnológico (CNPq), Chagas Disease Activities (CDIA)/ Comunidade Européia, convênio Secretaria de Vigilância em Saúde (SVS), Ministério da Saúde / FIOCRUZ.

\section{Referências Bibliográficas}

BARATA, J.M.S. 1981. Aspectos morfológicos de ovos de Triatominae II Características macroscópicas e exocoriais de dez espécies do gênero Rhodnius Stål 1859 (Hemiptera, Reduviidae). Rev. Saúde Pública, 15:490-542.

BARATA, J.M.S. 1998. Estruturas macroscópicas e exocoriais de ovos de Triatominae. In Atlas dos vetores da doença de Chagas nas Américas (R.U. Carcavallo, I. Galíndez Girón, J. Jurberg \& H. Lent, eds). Editora Fiocruz. Rio de Janeiro, p. 409-448. Vol. 2.

CARCAVALLO, R.U., JURBERG, J., LENT, H., NOIREAU, F. \& GALVÃO, C. 2000. Phylogeny of the Triatominae (Hemiptera, Reduviidae). Proposals for taxonomic arrangements. Entomol. Vect. 7(Suppl. 1):1-86.
CARCAVALLO, R.U., GALÍNDEZ-GIRÓN, I., JURBERG, J. \& LENT, H. 1998. Atlas of Chagas Disease vectors in the Américas. In Atlas dos vetores da doença de Chagas nas Américas (R.U. Carcavallo, I. Galíndez Girón, J. Jurberg \& H. Lent, eds). Editora Fiocruz. Rio de Janeiro, p. 311-393. Vol. 1.

CARCAVALLO, R.U., GALÍNDEZ-GIRÓN, I., JURBERG, J. \& LENT, H. 1999. Atlas of Chagas Disease vectors in the Américas. In Atlas dos vetores da doença de Chagas nas Américas (R.U. Carcavallo, I. Galíndez Girón, J. Jurberg \& H. Lent, eds). Editora Fiocruz. Rio de Janeiro, p. 07-733. Vol. 2 .

CARCAVALLO, R.U., GALÍNDEZ-GIRÓN, I., JURBERG, J. \& LENT, H. 1999. Atlas of Chagas Disease vectors in the Américas. In Atlas dos vetores da doença de Chagas nas Américas (R.U. Carcavallo, I. Galíndez Girón, J. Jurberg \& H. Lent, eds). Editora Fiocruz. Rio de Janeiro, p. 746-1217. Vol. 3.

GALVÃO, C., CARCAVALLO, R.U., ROCHA, D.S. \& JURBERG, J. 2003. A cheeklist of the current valid species of the subfamily Triatominae Jeannel, 1919 (Hemiptera, Reduviidae) and their geographycal distribution, with nomenclatural and taxonomic notes. Zootaxa, 202:1-36.

JURBERG, J. 1995. Uma abordagem filogenética entre os triatomíneos baseada nas estruturas fálicas. In Proceedings of the International Workshop on Population Genetics and Control of Triatominae (C.J. Schofield, J.P. Dujardin \& J. Jurberg, coord.). Inst. Nac. Diag Referência Epidemiológica, Santo Domingo de Los Colorados, p. 51-52.

JURBERG, J., LENT, H. \& GALVÃO, C. 1998. The male genitalia and its importance in taxonomy. Genitália dos machos e sua importância em Taxonomia. In Atlas of Chagas'disease vectors in the Américas. (R.U. Carcavallo, I. Galíndez Girón, J. Jurberg \& H. Lent, eds). Editora Fiocruz. Rio de Janeiro, p. 85-106. Vol. 1.

JURBERG, J. 1999. Noventa anos da descoberta da doença de Chagas e a criação do Centro de Referência em Taxonomia de Triatomíneos. Entomol. Vect. 6(4):315-322.

JURBERG, J., GALVÃO, C., NOIREAU, F., CARCAVAllo, R.U., ROCHA, D.S. \& LENT, H. 2005. Uma iconografia de Triatomíneos. Une iconographie des Triatomine. Editora Gama Filho, Rio de Janeiro. p. $1-49$.

JURBERG, J. \& GALVÃO, C. 2006. Biology, ecology and systematics of Triatominae (Heteroptera, Reduviidae) vectors of Chagas'disease and implications for human health. Denisia, 19:1096-1116.

LENT, H. \& JURBERG, J. 1969. O gênero Rhodnius Stål, 1859 com um estudo sobre a genitália das espécies (Hemiptera, Reduviidae, Triatominae). Rev. Brasil. Biol. 29(4):487-560.

LENT, H. \& WYGODZINSKY, P. 1979. Revision of the Triatominae and their significance as vectors of Chagas'disease. Bull. Am. Mus. Nat. History, 163(3):123-520.

LENT, H., JURBERG, J. \& GALVÃO, C. 1993. Rhodnius stali n. sp. afim de Rhodnius pictipes Stål, 1872 (Hemiptera, Reduviidae, Triatominae). Mem. Inst. Oswaldo Cruz, 88(4):605-614.

MEJIA, J.M., GALVÃO, C. \& JURBERG, J. 1999. Rhodnius colombiensis sp. n. da Colômbia com quadros comparativos entre as estruturas fálicas do gênero Rhodnius Stål 1859 (Hemiptera, Reduviidae, Triatominae). Entomol. Vect. 6(6):601-617.

SHERLOCK, I.A., GUITTON, N. \& MILLES, M. 1977. Rhodnius paraensis espécie nova do estado do Pará, Brasil (Hemiptera, Reduviidae, Triatominae). Act. Amaz. 7(1):71-74.

Recebido em 20/12/07

Versão reformulada recebida em 30/11/08

Publicado em 09/03/09 